\title{
Regional anaesthesia in neonates: a narrative review
}

\author{
V Ponde, (ID K Puri, (iD T Nagdev iD \\ Corresponding author, email: vrushaliponde@yahoo.co.in
}

\section{Summary}

The benefits of regional anaesthesia and the accompanying concerns of general anaesthesia in newborns make the former a safe and valuable addition. Multiple regional anaesthesia techniques, ranging from the central neuraxial blocks, truncal blocks, and peripheral nerve blocks are available. Empowering us to make the case to case decision to choose the most beneficial and safe block for the neonate. The applications of regional anaesthesia, over the period, have encompassed perioperative analgesia and therapeutic uses. With the addition of ultrasonography, neonatal regional anaesthesia has reached a fine amount of objectivity and accuracy. A trend of utilising regional blocks as the sole anaesthetic as against an adjuvant to general anaesthesia is setting in. In this review, we take a look at recent developments in this field in a generalised manner followed by dwelling into most of the commonly used blocks in daily practice. However, a detailed description of each block is beyond the scope of this review.

Keywords: regional anaesthesia, neonates, neuraxial blocks, truncal blocks, peripheral nerve blocks

\section{Introduction}

Amidst the growing concern of neurotoxicity associated with general anaesthesia (GA) in neonates, the benefits of regional anaesthesia (RA) seem to be accentuated. RA prevents or decreases the exposure to $\mathrm{GA}$, hastens recovery time and obviates the concerns regarding neurotoxicity. Prior literature has rested the case over the debate of neonatal pain concluding that they do perceive pain, ${ }^{1}$ necessitating analgesia. Owing to the superior quality of analgesia it offers, RA becomes the most obvious choice. With a fitting indication, any regional technique that is practised in adults can be administered to neonates.

This review explores the recent trends of RA techniques in neonates and analyses the paradigm shift while choosing the RA techniques.

\section{Methodology}

A comprehensive literature search was performed in September 2020 in MEDLINE, PubMed and Google Scholar to retrieve articles pertaining to neonatal regional anaesthesia. The keywords used were 'Central neuraxial, blocks, Neonates', 'Peripheral Nerve blocks,' 'truncal blocks in neonates' and 'Safety'. We shall consider unfolding various general aspects and then the most commonly performed blocks in neonates.

\section{How safe is regional anaesthesia in neonates?}

RA has a good safety profile in neonates. ${ }^{2}$ However, sufficient training and thorough knowledge are prerequisites for its safety. The risk of local anaesthetic (LA) systemic toxicity is known to be higher $^{3}$ and the overall risk is more in neonates. ${ }^{4}$

\section{Advantages of RA in newborns}

Surgery produces acute stress response causing autonomic, hormonal, metabolic, immunologic and inflammatory effects and impacts morbidity if not treated. This leads to delayed wound healing, hypoxia and respiratory distress. Long term repercussions include neurodevelopmental impairment, a negative shift in pain sensitivity, emotional, behavioural, and learning disabilities. RA is shown to inhibit these responses effectively. By improving circulation, it helps recovery in ischaemic bowel disease, improves gut motility, decreases the time for ventilator weaning and overall neonatal intensive care unit (NICU) stay. ${ }^{2}$ Opioids are an alternative mode of analgesia. Respiratory depression, hypotension, sedation, tolerance and hyperalgesia are its known side effects.

\section{Equipment for neonatal RA}

RA in neonates requires specific equipment. A 19 and 20 gauge epidural sets are appropriate for an epidural block or any continuous blocks. For subarachnoid block, $30 \mathrm{~mm}$ long $25 \mathrm{G}$ Quincke spinal needles are recommended. The $22 \mathrm{G} 30 \mathrm{~mm}$ neonatal lumbar puncture needle and Whitacre spinal needle serve as suitable alternatives. These styletted needles avoid seeding of the epidermal tissue into the spinal canal, potentially causing the formation of epidermoid tumours. For peripheral blocks, $25 \mathrm{~mm}$ long, 24 gauge. Stimuplex ${ }^{\circledR}$, insulated needle is suitable. High-frequency ultrasound (US) probe with a small footprint (hockey stick $7-13 \mathrm{MHz}$ ) is ideal. However, the standard footprint probe can also be used.

\section{General considerations}

Although awake blocks are performed, neonates are sedated or administered GA prior to administration of RA. While positioning, 
a well-supported head and neck without flexion ensure a patent airway conduit. Vigilant monitoring of vitals is imperative while performing blocks. The choice of sedation or GA or an awake block is a case-based decision. If an awake block is considered, the needle puncture area could be rendered insensate by application of LA creams.

\section{Central neuraxial blocks in neonates}

\section{Spinal block}

Due to advances in NICU care and infertility treatments, the survival of extremely preterm babies is rising explaining the surge of ex preemie neonates undergoing herniotomies among other surgeries. Anatomical pointers such as the intercristal line remain crucial. ${ }^{5}$ It crosses at the L5-S1 interspace at birth and later ascends to $L 5$ vertebra in infants. The spinal cord ends between $L 2$ and $L 3$ vertebrae in premature and in between $L 1$ and $L 2$ vertebrae in term infants whereas, the dural sac lies at $S 4$ level at birth and reaches the S2 level by the end of the first year. ${ }^{6}$ US assistance may be desirable for real-time visualisation of the dural sac, and conus medullaris. Spinal anaesthesia has a definite endpoint ${ }^{6}$ and thus a shorter learning curve. It has a promise of reducing but not eliminating postoperative apnoea. ${ }^{7}$

The recommended dose is $0.8-1 \mathrm{mg} / \mathrm{kg}$ or $0.08 \mathrm{ml} / \mathrm{kg}$ of $0.5 \%$ hyperbaric bupivacaine or ropivacaine. Ideally, specialised paediatric spinal needles should be used.

The short duration of the block without residual analgesic effects limits its use in bilateral herniotomies or longer surgeries. Haemodynamic changes due to spinal block are not profound in this age group. However, apnoea and respiratory arrests are known complication due to high spinal block. Spinal cord injury should be avoided at all costs, US can aid in assessing the level of conus medullaris.

Drugs and dosages of the commonly used LA in every day practise in neonates:

- Ropivacaine $0.2 \% 2 \mathrm{mg} / \mathrm{kg}$

- Bupivacaine $0.25 \% 2 \mathrm{mg} / \mathrm{kg}$

- Lignocaine with adrenaline $2 \% 5 \mathrm{mg} / \mathrm{kg}$

Volume depends on the site of the surgery:

- Inguinal hernia: $1 \mathrm{ml} / \mathrm{kg}$

- Circumcision: $0.5 \mathrm{ml} / \mathrm{kg}$

- Bilateral tenotomies: $0.5 \mathrm{ml} / \mathrm{kg}$

- Cystoscopy fulguration: $0.5 \mathrm{ml} / \mathrm{kg}$

\section{Epidural anaesthesia}

The caudal epidural block is the most extensively used technique in neonates with a relatively short learning curve and diverse indications. The spread of block caters to procedures from upper thorax to the lower limb. Conventionally, it is administered by palpation of the sacral hiatus via landmark technique in the lateral position with gentle flexion at the hip and knees. In this position, the posterior superior iliac spine (PSIS) and the sacral hiatus are presumed to form an equilateral triangle. Unlike spinal block, it has a subjective endpoint in the form of pop or give-away experienced by the anaesthesiologist when the needle pierces the sacrococcygeal membrane. Recently with the advent of US these anatomical relations are questioned. Mirjalili et al observed the vagaries in anatomical landmarks of caudal space with US. ${ }^{8}$ Moreover, US enables us to visualise important structures such as dural sac, floor and roof of the caudal epidural space (Figure 1). The tissue expansion and cranial spread of the LA

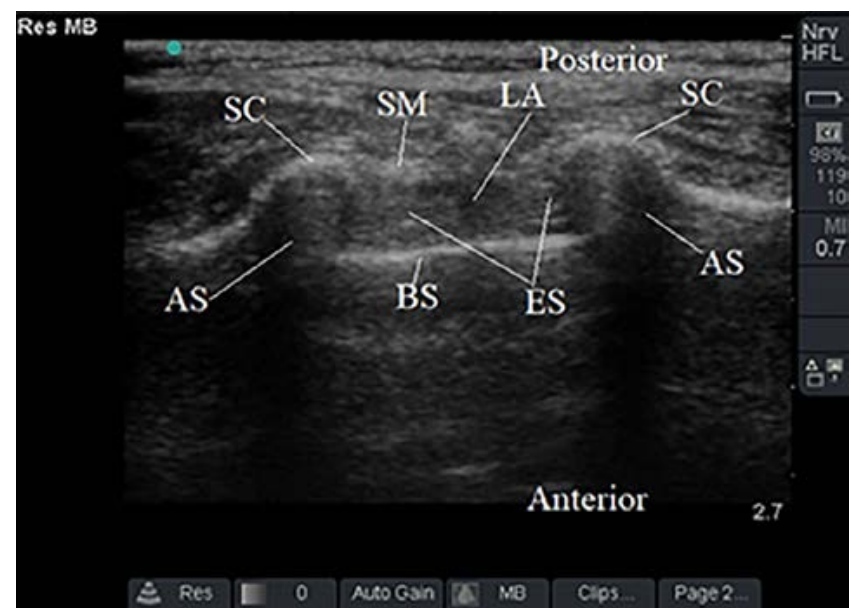

Figure 1: Transverse section at the level of sacral cornu in a neonate SC - sacral cornu, SM - sacrococcygeal membrane, ES - epidural space, AS - acoustic shadow, LA - local anaesthetic, BS - base of the sacrum

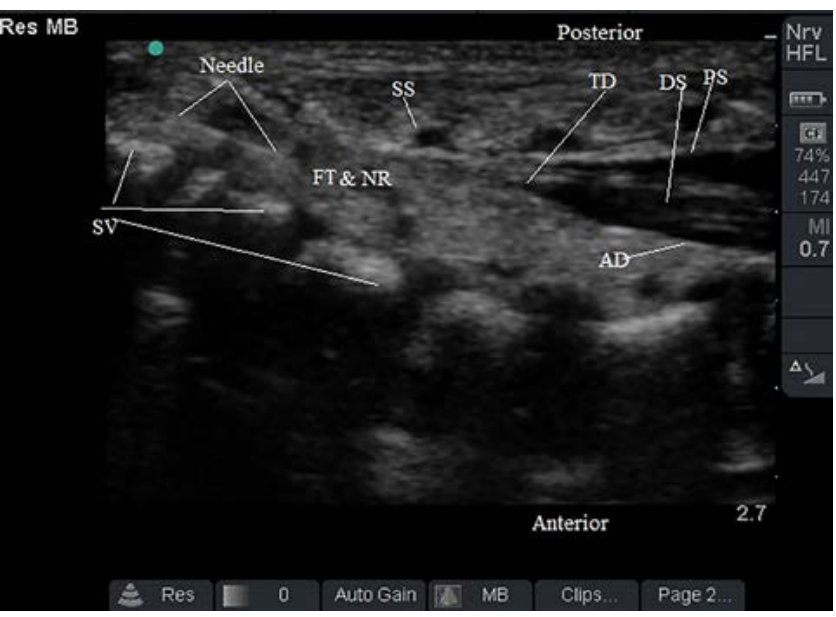

Figure 2: Longitudinal scan at the sacral level in a neonate

SS - sacral spine, TD - terminal of dural sac, DS - dural sac, PD - posterior dura $\mathrm{AD}$ - anterior dura, SV - sacral vertebra, FT - filum terminale, NR - nerve roots

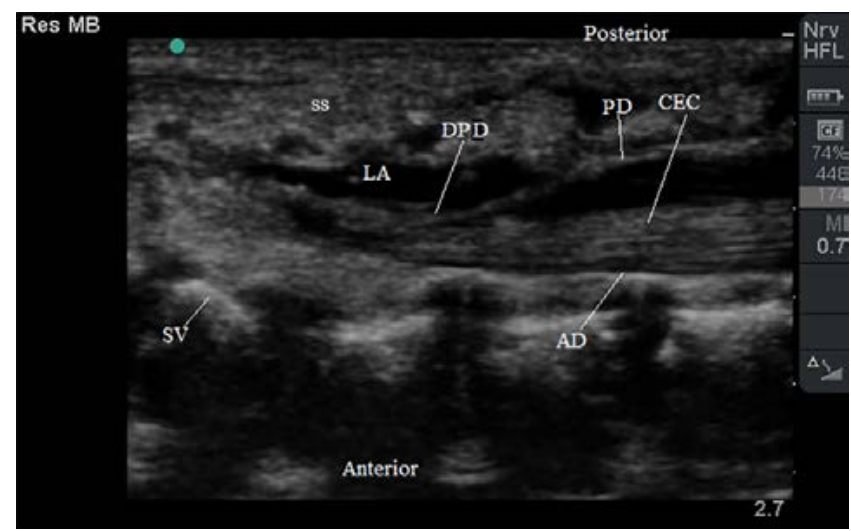

Figure 3: Longitudinal scan at the sacral level in a neonate, post drug injection

SS - sacral spine, LA - local anaesthetic, SV - sacral vertebra, DPD - displaced posterior dura, $\mathrm{PD}$ - posterior dura, $\mathrm{AD}$ - anterior dura 
(Figure 2 and 3) can be observed in real-time to ensure that the surgically congruent vertebral level is reached. ${ }^{9}$ Most importantly, US can pick up congenital anomalies which can influence the choice of RA technique itself. The epidural space median volumes are shown to be $1.30,1.57$, and $1.78 \mathrm{ml} / \mathrm{kg}$ at the L1, T10, and T6 levels, respectively. ${ }^{10}$ Justifying the time tested Armitage formula which suggests a volume of $0.5 \mathrm{ml} /$ $\mathrm{kg}$ for sacral, $1.0 \mathrm{ml} / \mathrm{kg}$ lumbar, and $1.25 \mathrm{ml} / \mathrm{kg}$ mid-thoracic dermatomes. ${ }^{10}$ Total spinal, local anaesthetic systemic toxicity, sacral osteomyelitis, localised infections and block failures are reported complications. ${ }^{11}$

Catheterisation of the epidural space is extremely favourable for major surgical procedures. Continuous epidural analgesia (CEA) is indicated for thoracic and abdominal surgery in newborns and performed under GA. Recently, continuous caudal infusion as a sole anaesthetic for hernia repair has been described. ${ }^{12}$ An 18-gauge intravenous cannula is inserted into the caudal epidural space, and a catheter is passed into the epidural space. Alternatively, 19- or 20-gauge epidural needles with their respective catheters can be used. The cephalad advancement of the catheter is usually smooth due to the absence of secondary curvatures in the neonatal spine. However, it should never be advanced against resistance as this might indicate that it is abutting against a nerve root or coiling around itself. The catheter tip can be placed at the lumbar or thoracic level in congruence with the surgical incision.

It has been shown that the catheter may not conform to externally assessed length. ${ }^{13}$ Fluoroscopy (involves exposure to dye and radiation), stimulating catheters (increases the cost) and ultrasonography can be used to identify the tip of the catheter. As the spine is not ossified in this age group, the neuraxial conduit, the epidural catheter trajectory and the tip can be visualised very clearly (Figure 4) and adjusted in real-time. The drug injection can further confirm the catheter tip, if not seen very convincingly. Skin dressing and tunnelling should protect

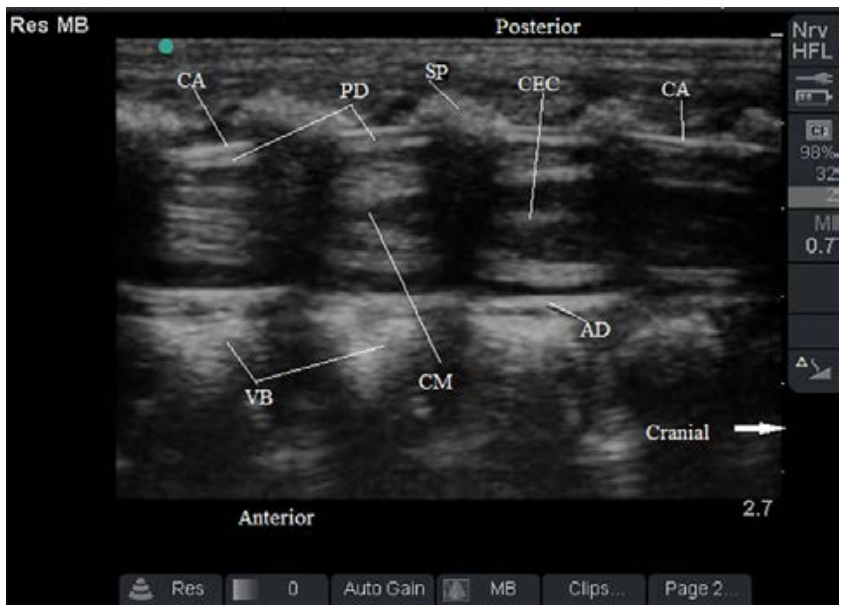

Figure 4: Longitudinal scan at the lumbar level in a neonate, post catheter via the caudal route

$\mathrm{CA}$ - catheter, PD - posterior dura, SP - spinous process, CEC - central echogenic complex of the anterior median fissure, $A D$ - anterior dura, $C M$ - conus medularis, VB - vertebral body them from contamination of stools as these are close to the perianal area.

Lumbar epidural, US guided or blind, is performed for major abdominal surgeries. The loss of resistance (LOR) is preferably obtained by saline rather than air. The risk of embolism, nerve root compression, patchy analgesia disfavours air. Conversely, while using saline for LOR, it may be mistaken for cerebrospinal fluid (CSF) or any drug. Thoracic epidural is described in neonates and should be strictly performed by an experienced neonatal anaesthesiologist. The risk of spinal cord injury against the benefit should be considered, especially when the catheter can be passed to the desired level by the caudal or lumbar route. Continuous infusion via catheter for 72 hours could be administered and the infusions should be labelled and the NICU team should be well versed with it.

\section{Truncal blocks}

Paravertebral (PVB) block is an alternative to the conventional central neuraxial RA technique, especially for unilateral thoracic surg-eries. ${ }^{14,15}$ In essence, the LA is injected in the wedge-shaped space adjacent to the vertebrae to block the spinal nerves as they emerge from the intervertebral foramen (Figure 5). The parietal pleura forms the anterolateral border, and posterior aspect of the paravertebal space is formed by the transverse processes (TPs) of the thoracic vertebrae, heads of the ribs, and the superior costotransverse ligament (Figure 5). This block is given under real-time US guidance, in longitudinal as well transverse scan. The authors prefer a transverse axis because of better visualisation of paravertebral spaces and their boundaries in newborns. Thompson and Haynes ${ }^{15}$ described an in-plane oblique intercostal approach for catheter placement with $0.08 \%$ ropivacaine infusion for $4-5$ days. They concluded that the block reduced opioid consumption and aided in weaning. The infusion dose was calculated at $0.2 \mathrm{mg} / \mathrm{kg}$ per hour which yielded an infusion rate of approximately $0.25 \mathrm{ml} / \mathrm{kg}$ per hour.

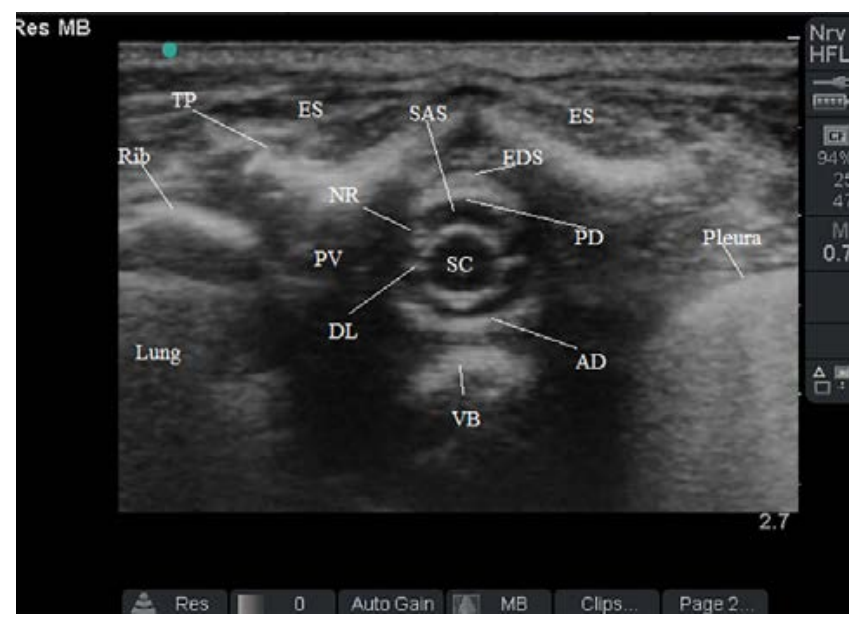

Figure 5: Transverse scan at the level of 6 th thoracic vertebral level $\mathrm{TP}$ - transverse process, ES - erector spinae, SAS - subarachnoid space, NR - nerve root, $\mathrm{PV}$ - paravertebral space, DL - denticulate ligament, VB - vertebral body, SC - spinal cord, AD - anterior dura, PD - posterior dura, EDS - epidural space 
Bigger series in infants have suggested equivalent analgesia for thoracotomy and reduced central side effects with landmarkbased paravertebral blocks (PVB).

\section{Erector spinae plane block}

ESPB is a surrogate to paravertebral block, promising unilateral analgesia and minimal complications. Technical ease and equivalent analgesia to the central neuraxial block is exhibited. This is an Interfascial plane block wherein the LA is injected in between the lateral tip of transverse process ${ }^{16}$ and the erector spinae (Figure 5). The LA is speculated to bathe the nerve roots exiting the respective level. A lot of research is on-going, literature in neonates is limited. The favourable outcome in the form of faster placement, good success rate and a reduced requirement of opioids was noted. A striking advantage of this block is its spread ranging from $\mathrm{T} 1$ to $L 4$, enabling it to be a handy block in a majority of neonatal surgeries.

\section{Transverses abdominis plane block}

Transverses abdominis plane (TAP) block is relatively confined but not limited to cases with coagulopathy or spinal abnormalities. This is a fascial plane block and the LA is injected under US guidance between the internal and transverses abdominis muscle. It is devoid of visceral analgesia, unlike the caudal epidural block. In a study by Kendigelen et al. ${ }^{17}$ US assisted TAP block was administered to 34 infants ( 2 to 88 day-old) undergoing supraumbilical (subcostal approach) and infraumbilical (midaxillary or posterior approach) surgeries. $0.5 \mathrm{ml} / \mathrm{kg}$ of $0.2 \%$ ropivacaine in the unilateral approach and $0.25 \mathrm{ml} / \mathrm{kg}$ of $0.25 \%$ bupivacaine on either side for bilateral block would be a safe approach.

\section{Rectus sheath block}

Although there are few studies of RSB in children, it is yet to be studied exclusively in neonates. It is indicated for analgesia of the umbilical port in laparoscopic surgeries. A US guided in-plane approach targeting the space between the rectus abdominis muscle and the posterior rectus sheath is suggested. This is usually found at a depth of $2 \mathrm{~mm}$ from the skin in this population. Peritoneal and bowel injury and haematoma can occur if this depth is exceeded.

\section{Upper extremity}

In routine practice, their applications seem to be more for the therapeutic sympatholytic procedures or for analgesia in fasciotomy surgeries secondary to compartment syndrome due to extravasation of vascular catheters, as elective indication is unlikely.

Drug volume $0.3 \mathrm{ml} / \mathrm{kg} 0.25 \%$ bupivacaine or $0.2 \%$ bupivacaine is recommended. Interscalene block, aiming the trunks, is indicated for shoulder and proximal arm surgeries, which are usually rare in this age group. Complications include phrenic nerve palsy, pneumothorax and accidental intra-arterial injection.
Supraclavicular block, targeting the divisions is used for arm, elbow and hand surgeries. The proximity of subclavian artery cautions against blind blocks and use of real-time visualisation to avoid inadvertent vascular injection is advised. An interesting excerpt by Hubbard et al. described the application of continuous catheter with this approach in a neonate with a gangrenous right upper extremity requiring multiple painful debridements over several weeks. ${ }^{18}$

A more distally administered approach is blocking of cords via infraclavicular block. Due to ease of catheter insertion and fixation, it is a preferred method. US guided infraclavicular block in limiting the area of gangrene to the digits secondary to inadvertent arterial puncture has been reported..$^{19}$

\section{Dorsal penile nerve block and ring block}

Circumcision is the commonest procedure in newborns and deserves a thoughtful approach towards analgesia. Alternatives such as EMLA cream application, ring block, dorsal penile nerve block in increasing order of technical expertise can be implemented. These blocks can be performed in newborns wrapped in a soft cloth, warm temperature and while they suck on a sucrose-soaked gauze piece.

Ring block is a simple technique of injecting LA in the subcutaneous tissue around the base of the penis, while it is gently held vertical. Alternatively, a dorsal penile nerve block can provide better analgesia, as compared to acetaminophen. The LA is deposited at the level of right and left dorsal nerves of the penis. With the penis gently retracted caudally, just below the symphysis pubis, the needle ( 27 gauge) is advanced perpendicularly to feel the give way of the Scarpa's fascia. LA is then injected at 10 and 2 o'clock position respectively after aspiration. The superficial penile vein may get punctured with this approach. Approximately, $0.5 \mathrm{ml}$ of ropivacaine $0.2 \%$ is sufficient for a newborn. This block can also be placed under ultrasound guidance. With a high-frequency transducer the subpubic space with its boundaries is visualised with the unmistakable pubic symphysis superiorly, the Scarpa fascia anteriorly, and the Buck's fascia posteriorly. Adequate time for LA action should be ensured if an awake technique is planned.

Lower limb blocks comprising of the sciatic and femoral block are not too common due to the easier access to the caudal epidural space and the requirement of two blocks to render analgesia for the lower extremity. However, they are useful with appropriate indications and in presence in coagulopathy.

The inferior alveolar nerve blocks have been described for neonates, ${ }^{20}$ this deserves a special mention. The report describes that the block was given for mandibular distraction and osteotomies in a newborn. This was shown to lower the pain scores with about 24 hours of analgesia duration. The authors report the application of serial blocks for pain control which seemed to be helpful in very painful surgeries such as these.

In conclusion, neonatal regional anaesthesia has a wide scope. Its astute understanding and application are shown to have increased safety and benefits. Over the years some techniques 
have proved the test of time and some are new on the horizon, with their efficacy awaited. However, this modality is here to stay and has come a long way.

\section{ORCID}

\section{Ponde (iD https://orcid.org/0000-0001-6300-7240 \\ K Puri (iD https://orcid.org/0000-0002-0917-9655}

\section{T Nagdev (iD https://orcid.org/0000-0003-4248-5993}

\section{References}

1. Marchant A. 'Neonates do not feel pain': a critical review of the evidence. Biosci Horizons. 2014;7:hzu006. https://doi.org/10.1093/biohorizons/hzu006.

2. Bosenberg A. Benefits of regional anesthesia in children. Pediatr Anesth. 2012;22(1):10-8. https://doi.org/10.1111/j.1460-9592.2011.03691.x.

3. Walker BJ, Long JB, Sathyamoorthy M, et al. Complications in pediatric regional anesthesia: an analysis of more than 100,000 blocks from the Pediatric Regional Anesthesia Network. Anesthesiology. 2018;129(4):721-32. https://doi. org/10.1097/ALN.0000000000002372.

4. Wong GK, Arab AA, Chew SC, et al. Major complications related to epidural analgesia in children: a 15-year audit of 3,152 epidurals. Can J Anesth. 2013;60:355-63. https://doi.org/10.1007/s12630-012-9877-3.

5. Van Schoor A, Bosman MC, Bosenberg AT. The value of Tuffier's line for neonatal neuraxial procedures. Clin Anat. 2014;27(3):370-5. https://doi.org/10.1002/ ca.22218.

6. Subramaniam R. Anaesthetic concerns in preterm and term neonates. Indian J Anaesth 2019;63(9):771-9. https://doi.org/10.4103/ija.IJA_591_19.

7. Jones LJ, Craven PD, Lakkundi A, Foster JP, Badawi N. Regional (spinal, epidural, caudal) versus general anaesthesia in preterm infants undergoing inguinal herniorrhaphy in early infancy. Cochrane Database Syst Rev. 2015;2015:CD003669. https://doi.org/10.1002/14651858.CD003669.pub2.

8. Mirjalili SA, Taghavi K, Frawley G, Craw S. Should we abandon landmark-based technique for caudal anesthesia in neonates and infants? Pediatric Anesthesia. 2015;25(5):511-6. https://doi.org/10.1111/pan.12576.

9. Lönnqvist PA. Regional anaesthesia and analgesia in the neonate. Best Pract Res Clin Anaesthesiol. 2010;24(3):309-21. https://doi.org/10.1016/j.bpa.2010.02.012.

10. Forestier J, Castillo P, Finnbogason, et al. Volumes of the spinal canal and caudal space in children zero to three years of age assessed by magnetic resonance imaging: implications for volume dosage of caudal blockade. $\mathrm{Br} J$ Anaesth 2017;119(5):972-8. https://doi.org/10.1093/bja/aex280.

11. Wiegele M, Marhofer P, Lönnqvist P-A. Caudal epidural blocks in paediatric patients: a review and practical considerations. Br J Anaesth. 2019;122(4):509-17 https://doi.org/10.1016/j.bja.2018.11.030.

12. Mueller CM, Sinclair TJ, Stevens M, Esquivel M, Gordon N. Regional block via continuous caudal infusion as sole anesthetic for inguinal hernia repair in conscious neonates. Pediatr Surg Int. 2017;33:341-5. https://doi.org/10.1007/ s00383-016-4027-6.

13. Ponde VC, Bedekar VV, Desai AP, Puranik KA. Does ultrasound guidance add accuracy to continuous caudal-epidural catheter placements in neonates and infants? Paediatr Anaesth. 2017;27(10):1010-4. https://doi.org/10.1111/ pan.13212.

14. Hernandez MA, Visoiu M. Postoperative pain management for tracheoesophageal fistula repair using transverse, in-plane, ultrasound guided paravertebral technique in a $2 \mathrm{~kg}$ neonate. J Clin Anesth. 2018:50:57-8. https:// doi.org/10.1016/j.jclinane.2018.06.041

15. Thompson ME, Haynes B. Ultrasound-guided thoracic paravertebral block catheter experience in 2 neonates. J Clin Anesth. 2015;27(6):514-6. https://doi. org/10.1016/j.jclinane.2015.05.002.

16. El-Morsy GZ, El-Deeb A, El-Desouky T, Elsharkawy AA, Elgamal MAF. Can thoracic paravertebral block replace thoracic epidural block in pediatric cardiac surgery? A randomized blinded study. Ann Card Anaesth. 2012;15(4):259-63. https://doi. org/10.4103/0971-9784.101848.

17. Kendigelen $P$, Tütüncü $C$, Ashyralyyeva $G$, et al. Transversus abdominis plane (TAP) block for postoperative analgesia in neonates and young infants: retrospective analysis of a case series. TAP blocks in neonates and young infants. Minerva Anestesiol. 2017;83(3);282-7. https://doi.org/10.23736/ s0375-9393.16.11420-8.

18. Hubbard RM, Cappuccio EC, Martin DP, et al. Ultrasound-guided, continuous brachial plexus blockade in a neonate with upper extremity limb ischemia: a case report. A A Pract. 2019;12(6):190-2. https://doi.org/10.1213/ XAA.0000000000000879.

19. Ponde VC, Shah DM, Mane S. Role of ultrasound-guided continuous brachial plexus block in the management of neonatal ischemia in upper limb. Saudi Anesth. 2012;6(4):423-5. https://doi.org/10.4103/1658-354X.105898.

20. Krodel DJ, Belvis $D$, Suresh $S$. Inferior alveolar nerve blocks for postoperative pain control after mandibular distraction with osteotomies in a neonate. Pediatr Anesth. 2014;24(6):635-7. https://doi.org/10.1111/pan.12379. 